\title{
Rad51 Regulates Reprogramming Efficiency through DNA Repair Pathway
}

\author{
Jae-Young Lee ${ }^{1}$, Dae-Kwan Kim ${ }^{1}$, Jeong-Jae Ko ${ }^{1}{ }^{\dagger} K_{\text {Keun Pil Kim }}{ }^{2}$ and ${ }^{\dagger}$ Kyung-Soon Park ${ }^{1}$ \\ ${ }^{1}$ Dept. of Biomedical Science, College of Life Science, CHA University, Seoul 06135, Korea \\ ${ }^{2}$ Dept. of Life Science, Chung-Ang University, Seoul 06975, Korea
}

\begin{abstract}
Rad51 is a key component of homologous recombination (HR) to repair DNA double-strand breaks and it forms Rad51 recombinase filaments of broken single-stranded DNA to promote HR. In addition to its role in DNA repair and cell cycle progression, Rad51 contributes to the reprogramming process during the generation of induced pluripotent stem cells. In light of this, we performed reprogramming experiments to examine the effect of co-expression of Rad51 and four reprogramming factors, Oct4, Sox2, Klf4, and c-Myc, on the reprogramming efficiency. Co-expression of Rad51 significantly increased the numbers of alkaline phosphatase-positive colonies and embryonic stem cell-like colonies during the process of reprogramming. Co-expression ofRad51 significantly increased the expression of epithelial markers at an early stage of reprogramming compared with control cells. Phosphorylated histone $\mathrm{H} 2 \mathrm{AX}(\gamma \mathrm{H} 2 \mathrm{AX})$, which initiates the DNA double-strand break repair system, was highly accumulated in reprogramming intermediates upon co-expression of Rad51. This study identified a novel role of Rad51 in enhancing the reprogramming efficiency, possibly by facilitating mesenchymal-to-epithelial transition and by regulating a DNA damage repair pathway during the early phase of the reprogramming process.
\end{abstract}

Key words : Rad51, Reprogramming, $\gamma \mathrm{H} 2 \mathrm{AX}$, Homologous recombination

\section{INTRODUCTION}

The ability to generate induced pluripotent stem cells (iPSCs) using a set of four defined transcription factors (Oct4, Sox2, Klf4, and c-Myc (OSKM)) provides great promise for generating patient-specific cells that are suitable for cell therapy, disease modeling, and drug screening (Chi et al., 2015; Tidball and Parent, 2016; Yamashita and Abe, 2016). Despite this great promise, reprogramming efficiencies in initial studies with the OSKM transcription factors were low $(0.01-0.02 \%)$ and are probably not sufficient for clinical applications (Ebrahimi, 2015).
Therefore, diverse strategies have been developed to improve the reprogramming efficiency, including the choice of various reprogramming factors, microRNAs, and small molecules and alteration of the culture conditions ( $\mathrm{Li}$ and Rana, 2012; Luni et al., 2016; Onder et al., 2012; Unternaehrer et al., 2014). These approaches target the main chromatinremodeling pathways as well as cellular signaling pathways.

Meanwhile, recent studies revealed that iPSCs generated by either integrative or non-integrative methods have genetic abnormalities (Gore et al., 2011; Hussein et al., 2011; Laurent et al., 2011). During the process of reprogramming, the levels of phosphorylated histone $\mathrm{H} 2 \mathrm{AX}(\gamma \mathrm{H} 2 \mathrm{AX})$, one of

\footnotetext{
Manuscript received May 18, 2016, Received in revised form May 29, 2016, Accepted June 09, 2016

${ }^{\dagger}$ Corresponding Author : Kyung-Soon Park, Dept. of Biomedical Science, College of Life Science, CHA University, Seoul 06135, Korea. Tel. : +82-31881-7144, E-mail : kspark@cha.ac.kr, Keun Pil Kim, Dept. of Life Science, Chung-Ang University, Seoul 06975, Korea. Tel. : +82-2-820-5792, E-mail: kpkim@cau.ac.kr

This is an Open Access article distributed under the terms of the Creative Commons Attribution Non-Commercial License (http:// creativecommons.org/licenses/by-nc/3.0) which permits unrestricted non-commercial use, distribution, and reproduction in any medium, provided the original work is properly cited.
} 
the earliest indictors of DNA double-strand breaks (DSBs), increase greatly, indicating that reprogramming is accompanied by severe genomic DNA damage (Gonzalez et al., 2013). Indeed, reprogramming factors induce apoptotic signals through the accumulation of oxidative stress and DNA damage (Banito et al., 2009; Kawamura et al., 2009). A study of the DNA repair mechanism during reprogramming of somatic cells into a pluripotent statefurther confirmed that the primary source of genetic instability is DNA DSBs that arise during the process of reprogramming (Gonzalez et al., 2013). DNA DSBs are repaired by homologous recombination (HR) or non-homologousend-joining (NHEJ). Defects in the major components of HR or NHEJ prevent the generation of iPSCs; therefore, an intact HR or NHEJ pathway is considered to be essential for efficient reprogramming (Felgentreff et al., 2014; Gonzalez et al., 2013).

$\operatorname{Rad} 51$ is a key component of HR and it forms $\operatorname{Rad} 51$ recombinase filaments of broken single-stranded DNA to promote HR (Sugawara et al., 2003; White and Haber, 1990). In addition, Rad51 regulates cell cycle progression in mouse embryonic stem cells by preserving G2/M transition (Yoon et al., 2014). Rad51 also facilitates replication fork progression by inhibiting replication fork collapse to ensure cell proliferation in vertebrates (Sonoda et al., 1998; Tsuzuki et al., 1996). Taken together, Rad51 is considered to play a critical role inprotecting genome integrity as well as resistance against apoptosis triggered by DNA damage both in stem cells and somatic cells. When expression of Rad51 is suppressed, the reprogramming efficiency is sig-nificantly decreased, indicating that Rad51 plays a critical role in reprogramming into a pluripotent state (Gonzalez et al., 2013).

In this study, we demonstrate that Rad51 enhances the reprogramming efficiency during the reprogramming of mouse embryonic fibroblasts (MEFs) into iPSCs. We further suggest that Rad51 facilitates the DNA damage response and mesenchymal-to-epithelial transition.

\section{MATERIALS AND METHODS}

\section{Reprogramming of MEFs}

MEFs expressing green fluorescent protein (GFP) from the Oct4 locus (OG-MEFs, passage 2) were purchased (Stemgent, MA, USA). To induced pluripotency, $4 \times 10^{5}$ cells were seeded on a $15 \mathrm{~cm}$ cell culture dish coated with $0.2 \%$ gelatin. The cells were transduced with pMx-based retroviral reprogramming factors. Twenty hours after transduction, the cells were transferred to J1 mouse embryonic stemcell growth media and maintained as described previously (Jirmanova et al., 2002).

\section{RNA extraction and real-time RT-PCR}

Total RNA was extracted using TRIzol (Invitrogen, CA, USA), and 2-5 $\mu \mathrm{g}$ of total RNA was reverse-transcribed using the SuperScriptIITM First-Strand Synthesis System (Invitrogen) according to the manufacturer's instructions. Real-time RT-PCR was performed using cDNA with the QuantiTectSYBR Green PCR Kit (Qiagen, CA, USA). Reactions were performed in triplicate using an Exicycler ${ }^{\mathrm{TM}} 96$ real-time quantitative thermal block (Bioneer, Daejon, Korea). For quantification, the expression levels of the target genes were normalized against those of the glyceraldehyde 3phosphate dehydrogenase gene.

\section{Protein extraction and immunoblotting}

Whole-cell extracts were prepared, and 20-50 $\mu \mathrm{g}$ of protein was resolved by SDS-PAGE and blotted using antibodies against Rad51 (sc-8349; Santa Cruz, CA, USA), $\gamma \mathrm{H} 2 \mathrm{AX}$ (ab22551; Abcam, CA, USA) and $\beta$-actin (sc-47778, Santa Cruz). Immunoreactivity was detected by enhanced chemiluminescence (Amersham, Buckinghamshire, England).

\section{Statistical analysis}

Graphical data are presented as means \pm standard deviation. Each experiment was performed at least three times 
and subjected to statistical analysis. Statistical significance between two groups was determined using the Student's $t$ test. A $p$ value less than 0.05 was considered significant. Statistical analysis was performed using the SAS statistical package v.9.13 (SAS Inc, NC, USA).

\section{RESULTS AND DISCUSSION}

To examine the effect of Rad51 expression on the reprogramming efficiency of MEFs, we co-transduced a Rad51- expressing retrovirus with four reprogramming factors (OSKM) and compared the number of alkaline phosphatase-positive $(\mathrm{AP}(+))$ colonies at 21 days after reprogramming initiation. Co-expression of Rad51 increased the number of $\mathrm{AP}(+)$ colonies by approximately 3.5-fold compared with OSKM transduction alone (Fig. 1A). To further confirm that Rad51 enhances the reprogramming of MEFs, we reprogrammed reporter MEFs expressing GFP under the control of the Oct4 promoter (OG-MEFs). Consistent with the results of alkaline phosphatasestaining, co-expression of Rad51 and

(A)

(B)
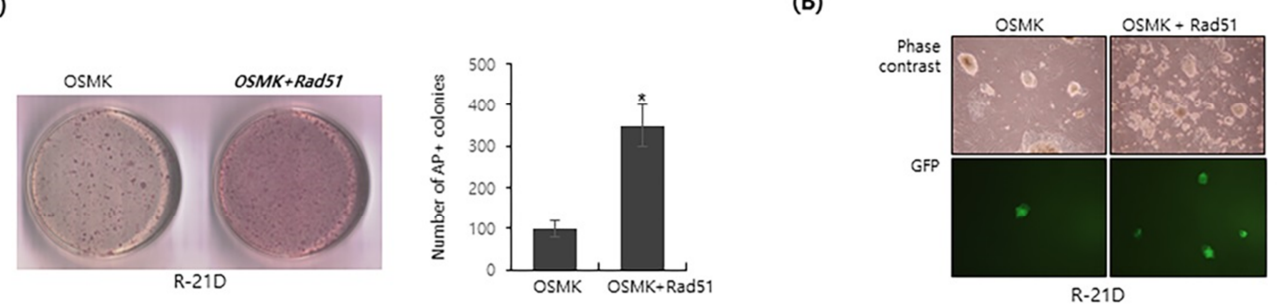

(C)
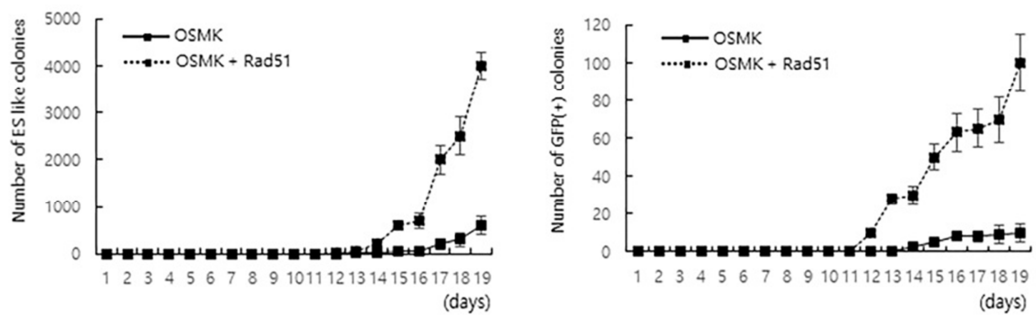

(D)
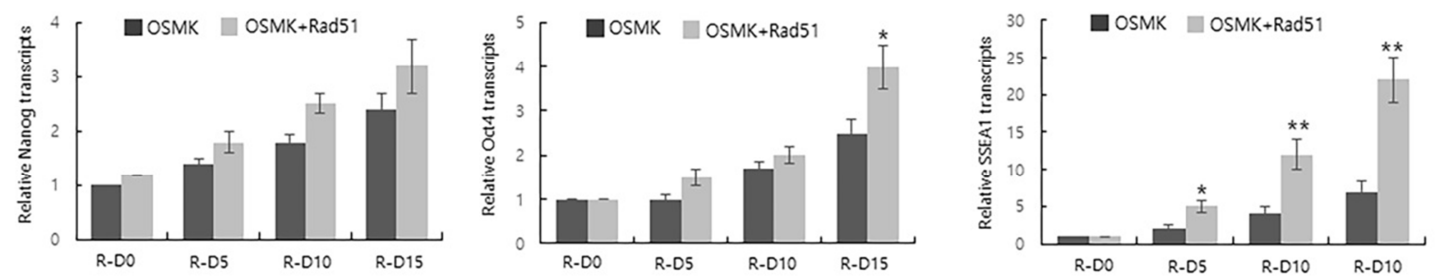

Fig. 1. Rad51 enhances the reprogramming efficiency of MEFs. (A) Alkaline phosphatase staining of iPSC colonies derived from MEFs. MEFs $\left(1 \times 10^{4}\right)$ were reprogrammed with OSKM or OSKM plus Rad51. AP $(+)$ colonies were counted at 21 days after the transduction of reprogramming factors. (B) Reprogramming of OG-MEFs. OG-MEFs $\left(1 \times 10^{4}\right)$ were reprogrammed with OSKM or OSKM plus Rad51. Colonies were observed under a fluorescence microscope at 21 days after the transduction of reprogramming factors. (C) ES-like colonies (left panel) and GFPpositive colonies (right panel) were counted at the indicated time points after reprogramming of OG-MEFs with OSKM or OSKM plus Rad51. (D) Expression of Nanog, Oct4 and SSEA1 was analyzed by real-time RT-PCR in reprogramming intermediates at the indicated time points after transduction of OSKM or OSKM plus Rad51. All values are means \pm standard deviation of at least three independent experiments. ${ }^{*} P<0.05$ and ${ }^{* *} P<0.01$ based on the Student's $t$-test. 
OSKM enhanced the number of colonies expressing GFP, which is a marker of activation of the Oct4 promoter by reprogramming (Fig. 1B). We next counted the numbers of ES-like colonies and GFP-expressing colonies during the reprogramming of OG-MEFs to examine whether Rad51 expression facilitates the reprogramming process. GFPpositive colonies as well as ES-like colonies appeared at least 3-4 days earlier when $\operatorname{Rad51}$ was co-expressed with OSKM (Fig. 1C). Even though Nanog expression was not significantly increased in the presence of Rad51 until 15 days after the initiation of reprogramming, the expression of Oct4 and SSEA1 was higher when Rad51 was coexpressed with reprogramming 4 factors (Fig. 1D). These results suggest that co-expression of Rad51and OSKM can facilitate the reprogramming process and enhance the reprogramming efficiency of MEFs.

Reprogramming is a process of mesenchymal-to-epi- thelial transition, which isaccompanied by upregulation of epithelial factors and down regulation of mesenchymal factors (Samavarchi-Tehrani et al., 2010). Therefore, we investigated the effect of Rad51 on the expression of epithelial and mesenchymal markers during the process of reprogramming. As expected, expression of Snail, a mesenchymal marker, was decreased, whereas expression of epithelial markers such as E-cadherin and epithelial cell adhesion molecule (Ep-CAM) was increased at the early stage of reprogramming (Fig. 2A). Compared with OSKM transduction alone, coexpression of Rad51 significantly increased E-cadherin and Ep-CAM expression from 6 days after reprogramming initiation, even though Rad51 expression did not have any effect on the expression of Snail (Fig. 2A).

Rad51 is a DNA repair protein that mediatesHR of DNA DSBs; therefore, we next examined whether co-expression of Rad51 modulates the DNA damage repair system during
(A)

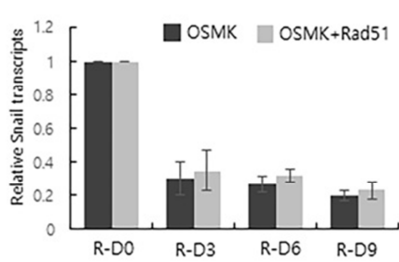

(B)

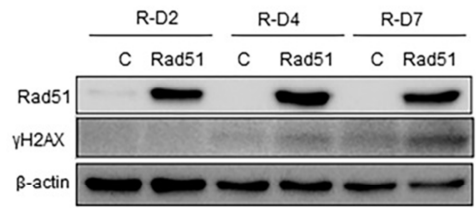

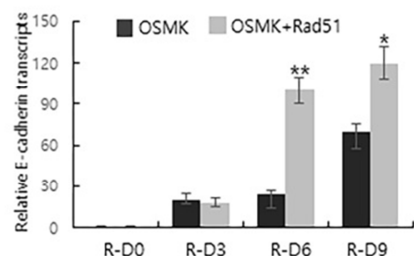

(C)

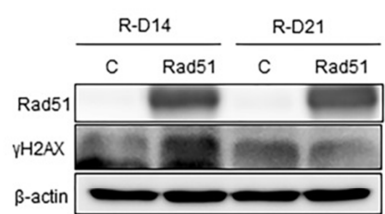

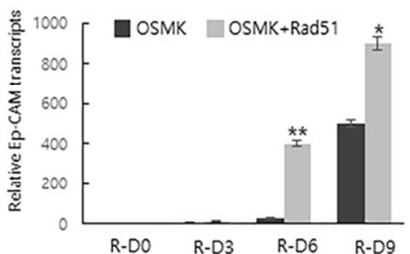

(D)

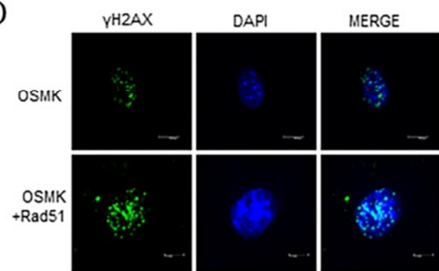

Fig. 2. Rad51 facilitates the DNA damage response during reprogramming. (A) Expression of Snail, E-cadherin, and Ep-CAM was analyzed by real-time RT-PCR in reprogramming intermediates at the indicated time points after transduction of OSKM or OSKM plus Rad51. Expression of $\gamma \mathrm{H} 2 \mathrm{AX}$ was analyzed by immunoblotting of reprogramming intermediates at the early $(\mathrm{B})$ or late $(\mathrm{C})$ phases of reprogramming. MEFs transduced with OSKM or OSKM plus Rad51 were analyzed at the indicated time points. (D) Expression of $\gamma \mathrm{H} 2 \mathrm{AX}$ was analyzed by immunocytochemical staining of MEFs reprogrammed with OSKM or OSKM plus Rad51 for 14 days. All values are means \pm standard deviation of at least three independent experiments. ${ }^{*} P<0.05$ and ${ }^{* *} P<0.01$ based on the Student's $t$-test. 
reprogramming. Phosphorylation of histone H2AX is essential for promoting DNA DSB repair by initiating the repair system (Kinner et al., 2008). Therefore, we compared the level of $\gamma \mathrm{H} 2 \mathrm{AX}$ in the presence and absence of Rad51 during the process of reprogramming. The level of $\gamma \mathrm{H} 2 \mathrm{AX}$ was increased in the presence of Rad51 during the early stage of reprogramming (Fig. 2B). To further confirm that $\gamma \mathrm{H} 2 \mathrm{AX}$ accumulates, we examined the level of $\gamma \mathrm{H} 2 \mathrm{AX}$ at 14 and 21 days after reprogramming initiation. The level of $\gamma \mathrm{H} 2 \mathrm{AX}$ was highly increased after 14 days of reprogramming in the presence of Rad51 (Fig. 2C). Immunocytochemical staining further showed that the level of $\gamma \mathrm{H} 2 \mathrm{AX}$ was high after 14 days of reprogramming (Fig. 2D). Taken together, we concluded that the ability of Rad51 to enhance the reprogramming efficiency is associated with promotion of the DNA repair mechanism during the reprogramming process.

In this study, we provide compelling evidence that Rad51 plays an important role inincreasing the reprogramming efficiency of MEFs. The mutation rate of pluripotent stem cells are relatively low compared with somatic cells at least in part by the active operation of HR pathway to repair DNA damage (Chlon et al., 2016). The molecular mechanisms by which Rad51 activity enhances the reprogramming efficiency remain obscure, although we speculate that the primary mechanism is activation of the DNA damage response. Indeed, $\gamma \mathrm{H} 2 \mathrm{AX}$ began to accumulate early during reprogramming when Rad51was co-expressed with OSKM. This result indirectly indicates that expression of Rad51 facilitates activation of the HR repair system.

Another possible mechanism by which Rad51 activity enhances the reprogramming efficiency is through regulation of the cell cycle. Cell cycle progression is closely associated with the efficiency of reprogramming, and the reprogramming factor c-Myc plays a central role inestablishing the pluripotent cell-specific cell cycle structure (Singh and Dalton, 2009).

In addition to its activity in HR, Rad51preserves cell cycle progression by preventing DNA replication fork collapse and mediates replication restart by functioning at stalled replication forks (Somyajit et al., 2015). Given that the reprogramming process isaccompanied by severe replication stress, it is also reasonable to hypothesize that Rad51 might be able to facilitate cell cycle progression by reducing replication stress during the reprogramming process.

The genomic instability of iPSCs has raised concerns about their clinical use; therefore, it is essential to determine whether co-expression of Rad51 and OSKM increases the genomic stability of established iPSCs, in addition to enhancing the reprogramming efficiency.

\section{ACKNOWLEDGEMENTS}

This work was performed with the support of the Cooperative Research Program for Agriculture Science \& Technology Development (PJ010033), Rural Development Administration, Republic of Korea. This work was also supported by the Korea Science and Engineering Foundation (KOSEF) of the Korean government (MOST) (2012M3A9 C6050367).

\section{REFERENCES}

Banito A, Rashid ST, Acosta JC, Li S, Pereira CF, Geti I, Pinho S, Silva JC, Azuara V, Walsh M, Vallier L, Gil J (2009) Senescence impairs successful reprogramming to pluripotent stem cells. Genes Dev 23:2134-2139.

Cano A, Perez-Moreno MA, Rodrigo I, Locascio A, Blanco MJ, del Barrio MG, Portillo F, Nieto MA (2000) The transcription factor snail controls epithelial-mesenchymal transitions by repressing E-cadherin expression. Nat Cell Biol 2:76-83.

Chi HJ, Gao S, Yang XC, Cai J, Zhao WS, Sun H, Geng YJ (2015) Clinical application of induced pluripotent stem cells in cardiovascular medicine. Cardiology 131: 236-244.

Chlon TM, Ruiz-Torres S, Maag L, Mayhew CN, Wikenheiser- 
Brokamp KA, Davies SM, Mehta P, Myers KC, Wells JM, Wells SI (2016) Overcoming pluripotent stem cell dependence on the repair of endogenous DNA damage. Stem Cell Reports 6:44-54.

Ebrahimi B (2015) Reprogramming barriers and enhancers: strategies to enhance the efficiency and kinetics of induced pluripotency. Cell Regen (Lond) 4:10.

Felgentreff K, Du L, Weinacht KG, Dobbs K, Bartish M, Giliani S, Schlaeger T, DeVine A, Schambach A, Woodbine LJ, Davies G, Baxi SN, van der Burg M, Bleesing J, Gennery A, Manis J, Pan-Hammarstrom Q, Notarangelo LD (2014) Differential role of nonhomologous end joining factors in the generation, DNA damage response, and myeloid differentiation of human induced pluripotent stem cells. Proc Natl Acad Sci U S A 111:8889-8894.

Gonzalez F, Georgieva D, Vanoli F, Shi ZD, Stadtfeld M, Ludwig T, Jasin M, Huangfu D (2013) Homologous recombination DNA repair genes play a critical role in reprogramming to a pluripotent state. Cell Rep 3:651660.

Gore A, Li Z, Fung HL, Young JE, Agarwal S, AntosiewiczBourget J, Canto I, Giorgetti A, Israel MA, Kiskinis E, Lee JH, Loh YH, Manos PD, Montserrat N, Panopoulos AD, Ruiz S, Wilbert ML, Yu J, Kirkness EF, Izpisua Belmonte JC, Rossi DJ, Thomson JA, Eggan K, Daley GQ, Goldstein LS, Zhang K (2011) Somatic coding mutations in human induced pluripotent stem cells. Nature 471:63-67.

Hussein SM, Batada NN, Vuoristo S, Ching RW, Autio R, Narva E, Ng S, Sourour M, Hamalainen R, Olsson C, Lundin K, Mikkola M, Trokovic R, Peitz M, Brustle O, Bazett-Jones DP, Alitalo K, Lahesmaa R, Nagy A, Otonkoski T (2011) Copy number variation and selection during reprogramming to pluripotency. Nature 471:5862.

Kawamura T, Suzuki J, Wang YV, Menendez S, Morera
LB, Raya A, Wahl GM, Izpisua Belmonte JC (2009) Linking the p53 tumour suppressor pathway to somatic cell reprogramming. Nature 460:1140-1144.

Kinner A, Wu W, Staudt C, Iliakis G (2008) Gamma$\mathrm{H} 2 \mathrm{AX}$ in recognition and signaling of DNA doublestrand breaks in the context of chromatin. Nucleic Acids Res 36:5678-5694

Kwon YW, Jang S, Paek JS, Lee JW, Cho HJ, Yang HM, Kim HS (2015) E-Ras improves the efficiency of reprogramming by facilitating cell cycle progression through JNK-Sp1 pathway. Stem Cell Res 15:481-494.

Laurent LC, Ulitsky I, Slavin I, Tran H, Schork A, Morey R, Lynch C, Harness JV, Lee S, Barrero MJ, Ku S, Martynova M, Semechkin R, Galat V, Gottesfeld J, Izpisua Belmonte JC, Murry C, Keirstead HS, Park HS, Schmidt U, Laslett AL, Muller FJ, Nievergelt CM, Shamir R, Loring JF (2011) Dynamic changes in the copy number of pluripotency and cell proliferation genes in human ESCs and iPSCs during reprogramming and time in culture. Cell Stem Cell 8:106-118.

Li Z, Huang H, Chen P, He M, Li Y, Arnovitz S, Jiang X, He C, Hyjek E, Zhang J, Zhang Z, Elkahloun A, Cao D, Shen C, Wunderlich M, Wang Y, Neilly MB, Jin J, Wei M, Lu J, Valk PJ, Delwel R, Lowenberg B, Le Beau MM, Vardiman J, Mulloy JC, Zeleznik-Le NJ, Liu PP, Zhang J, Chen J (2012a) miR-196b directly targets both HOXA9/MEIS1 oncogenes and FAS tumour suppressor in MLL-rearranged leukaemia. Nat Commun $3: 688$.

Li Z, Li Y, Yi Y, Huang W, Yang S, Niu W, Zhang L, Xu Z, Qu A, Wu Z, Xu T (2012b) Dissecting a central flipflop circuit that integrates contradictory sensory cues in C. elegans feeding regulation. Nat Commun 3:776.

Li Z, Rana TM (2012) A kinase inhibitor screen identifies small-molecule enhancers of reprogramming and iPS cell generation. Nat Commun 3:1085.

Liu D, Zhang W, Mou D, He J, Ou YB, Wang QY, Li Z, 
Wang L, Zhao L, He S, Peng Y, Liu X, Chen C, Yu L, Liu G, Dong X, Zhang J, Chen C, Xu Z, Hu J, Chen X, Ma X, Xue Q, Zhou XJ (2012) Electronic origin of high-temperature superconductivity in single-layer FeSe superconductor. Nat Commun 3:931.

Luni C, Giulitti S, Serena E, Ferrari L, Zambon A, Gagliano O, Giobbe GG, Michielin F, Knobel S, Bosio A, Elvassore N (2016) High-efficiency cellular reprogramming with microfluidics. Nat Methods 13:446-452.

Onder TT, Kara N, Cherry A, Sinha AU, Zhu N, Bernt KM, Cahan P, Marcarci BO, Unternaehrer J, Gupta PB, Lander ES, Armstrong SA, Daley GQ (2012) Chromatinmodifying enzymes as modulators of reprogramming. Nature 483:598-602.

Samavarchi-Tehrani P, Golipour A, David L, Sung HK, Beyer TA, Datti A, Woltjen K, Nagy A, Wrana JL (2010) Functional genomics reveals a BMP-driven mesenchymalto-epithelial transition in the initiation of somatic cell reprogramming. Cell Stem Cell 7:64-77.

Singh AM, Dalton S (2009) The cell cycle and Myc intersect with mechanisms that regulate pluripotency and reprogramming. Cell Stem Cell 5:141-149.

Somyajit K, Saxena S, Babu S, Mishra A, Nagaraju G (2015) Mammalian RAD51 paralogs protect nascent DNA at stalled forks and mediate replication restart. Nucleic Acids Res 43:9835-9855.

Sonoda E, Sasaki MS, Buerstedde JM, Bezzubova O, Shinohara A, Ogawa H, Takata M, Yamaguchi-Iwai Y, Takeda S (1998) Rad51-deficient vertebrate cells accumulate chromosomal breaks prior to cell death. EMBO J 17: 598-608.

Sugawara N, Wang X, Haber JE (2003) In vivo roles of Rad52, Rad54, and Rad55 proteins in Rad51-mediated recombination. Mol Cell 12:209-219.

Tidball AM, Parent JM (2016) Concise review: Exciting cells: Modeling genetic epilepsies with patient-derived induced pluripotent stem cells. Stem Cells 34:27-33.

Tsuzuki T, Fujii Y, Sakumi K, Tominaga Y, Nakao K, Sekiguchi M, Matsushiro A, Yoshimura Y, MoritaT (1996) Targeted disruption of the Rad51 gene leads to lethality in embryonic mice. Proc Natl Acad Sci U S A 93:6236-6240.

Unternaehrer JJ, Zhao R, Kim K, Cesana M, Powers JT, Ratanasirintrawoot S, Onder T, Shibue T, Weinberg RA, Daley GQ (2014) The epithelial-mesenchymal transition factor SNAIL paradoxically enhances reprogramming. Stem Cell Reports 3:691-698.

White CI, Haber JE (1990) Intermediates of recombination during mating type switching in Saccharomyces cerevisiae. EMBO J 9:663-673.

Yamashita T, Abe K (2016) Recent progress in cell reprogramming technology for cell transplantation therapy. Neurol Med Chir (Tokyo) 56:97-101.

Yoon SW, Kim DK, Kim KP, Park KS (2014) Rad51 regulates cell cycle progression by preserving $\mathrm{G} 2 / \mathrm{M}$ transition in mouse embryonic stem cells. Stem Cells Dev 23:2700-2711. 